\title{
In silico identification of candidate microRNAs and their targets in potato somatic hybrid Solanum tuberosum (+) S. pinnatisectum for late blight resistance
}

\author{
Ritu Singh', Jagesh Kumar Tiwari*, Shashi Rawat', Vinay Sharma² and Bir Pal Singh ${ }^{1}$ \\ ${ }^{1}$ ICAR-Central Potato Research Institute, Shimla, Himachal Pradesh - 171 001, India \\ ${ }^{2}$ Department of Bioscience and Biotechnology, Banasthali University, Rajasthan - 304022, India
}

\section{*Corresponding author: jageshtiwari@gmail.com}

\begin{abstract}
We employed computational tools to identify candidate miRNAs from 153 putative known genes obtained from a previous microarray experiment by Singh et al. (2016) for late blight resistance. In this study, we identified eight miRNAs families (miR395, $821,1030,1510,2673,3979,5021$ and 5213) to the nine potato genes. Mature miRNAs located at different positions of pre-miRNAs are varied from 19 to 21 nucleotides in length. Of the total 343 published miRNAs in potato (miRBase), all identified miRNAs family are new to potato crop except miR395. In particular, these novel miRNAs were identified for pathogen-induced late blight resistance mechanism in interspecific potato somatic hybrid. Majority of the predicted target genes (Table 2) of these miRNAs are involved in different biological functions, including disease resistance proteins (NBS-LRR domains) and transcription factors families. This study offers an insight to identify potential candidate miRNAs and their targets to dissect late blight resistance mechanism in potato somatic hybrid Solanum tuberosum (+) S. pinnatisectum.
\end{abstract}

Keywords: In silico, late blight resistance, miRNA, potato somatic hybrid, targets. Abbreviations: miRNAs: MicroRNAs.

\section{Introduction}

MicroRNAs (miRNAs) are extensive class of small, endogenous, single-stranded, short length $(\sim 19-24 \mathrm{nt})$ RNA molecules that bind to the 3 ' untranslated regions or to the open reading frames of target gene. In the both plants and animals, miRNAs negatively regulate gene expression at the post-transcriptional levels by binding to target mRNAs for initiating mRNA cleavage or inhibition of mRNA translation. In particular, miRNAs play essential roles in various plant developmental processes and during vast array of biotic and abiotic stresses such as cold, drought, salinity, nutrient deficiency and oxidative stress (Chen, 2009). With the discovery of first miRNAs (lin-4 and let-7) in Caenorhabditis elegans (Olsen et al., 1999), in plants first miRNA was identified in Arabidopsis thaliana in 2002 (Park et al., 2002). Since then with the advent of modern computational tools and conserved nature of mature miRNAs, several miRNAs have been identified across the plant species such as potato (Xie et al., 2011; Yang et al., 2013; Zhang et al., 2013), Solanaceae plants (Kim et al., 2011), Arabidopsis (Liang et al., 2015), rice (Campo et al., 2013), wheat (Akdogan et al., 2015), Brassica juncea (Srivastava et al., 2013), chickpea (Kohli et al., 2014), citrus (Lu et al., 2014) and peach (Luo et al., 2013) to name a few. To date, a total of 24,521 distinct mature hairpin precursor miRNAs across 206 species have been annotated in the miRBase Release 20 , an open online repository. There are various approaches currently been used to identify miRNAs such as genetic screening, direct cloning, computational strategy, ESTs and deep sequencing technology (Zhang et al., 2013). Identification of potential miRNA targets is crucial to understand function, which depends on the target gene expression. In potato (Solanum tuberosum L.) 343 published miRNAs (miRBase Release 20, June 2013) have been reported by a computational approach using available ESTs, genomic survey sequence and nucleotide databases. Zhang et al. (2013) identified of 120 potato-specific miRNA families and 28 conserved miRNA families by high-throughput sequencing of leaf and stolon tissues. However, no work has been reported on identification of miRNAs and their targets in interspecific potato somatic hybrid. In this study, we identified candidate miRNAs and their targets by computational approaches from previously identified 153 up-regulated transcripts for late blight resistance with known putative functions by microarray (Singh et al. 2016). Late blight caused by the oomycete Phytophthora infestans (Mont.) de Bery is the most devastating disease of potato worldwide (Tiwari et al., 2015). This work may increase our knowledge to understand molecular mechanism underlying resistance to late blight in our somatic hybrid. Although, conserved miRNAs have been known across the crop species for various plant development metabolism and stress response, none of them were known before for potato late blight resistance mechanism. To our limited knowledge on miRNAs this may be the first ever report on identification of novel conserved miRNAs candidates in potato somatic hybrid for late blight resistance.

\section{Results and Discussion}

Identification of miRNAs

In the plant kingdom most of the mature miRNAs are evolutionarily conserved from species to species, providing a 
powerful approach to predict the existence of new miRNA homologs in other plant species. We applied a homologybased BLASTn miRNA search with the known mature miRNAs sequences of plant species for our 153 up-regulated transcript sequences identified for late blight resistance in potato somatic hybrid $S$. tuberosum (+) S. pinnatisectum. A total of 16 miRNAs (stu-miR395x; stu-miR821b; stumiR1030a, stu-miR1030b, stu-miR1030c, stu-miR1030d, stumiR1030e, stu-miR1030f and stu-miR1030g; stu-miR1510a and stu-miR1510b; stu-miR2673a and stu-miR2673b; stumiR3979-5p; stu-miR5021; and stu-miR5213) belonging to eight miRNAs families (miR395, 821, 1030, 1510, 2673, 3979,5021 and 5213) were identified to the total nine potato genes (Table 1). Of the 343 mature miRNAs known in potato, only miR395 is known in potato, rest seven identified miRNAs families are new to the potato crop. In fact, all eight miRNAs families comprising 16 conserved miRNAs in this study are novel for late blight resistance in potato somatic hybrid. Phylogentic tree showing genetic diversity among the identified eight miRNAs sequences are shown in Fig. 1.

Among the eight identified miRNAs families, miR1030 was the largest family with seven members (stu-miR1030a to stumiR1030g) and identified to four potato genes (PGSC0003DMG 400036193, PGSC0003DMG400037942, PGSC0003DMG 400045212 and PGSC0003DMG 400040435). The stu-miR1510 (stu-miR1510a and stumiR1510b) and stu-miR5213 families were identified to gene PGSC0003DMG400026433. The miRNAs families namely stu-miR2673 (2 members), stu-miR3979-5p and stu-miR5021 were identified to gene PGSC0003DMG400038875. Whereas, three miRNAs families (stu-miR395x, stu-miR821b and stu-miR2673) were identified to genes PGSC0003DMG405025785, PGSC0003DMG 400019806 and PGSC0003DMG400021883, respectively (Table 1). PremiRNA sequences of this miRNAs were diverse in both structure and size. All 16 miRNAs sequences were varied between 19-21 nt and located at the $5^{\prime}$ arm of stem-loop hairpin of pre-miRNA sequences, which is similar to other variable length of miRNAs in potato (Xie et al., 2011; Zhang et al., 2009).

The stabilization of RNA secondary structure was based on the composition of four nucleotides (A, G, C and $\mathrm{U}$ ) in premiRNAs that require higher total $\mathrm{A}$ and $\mathrm{U}$ contents as compared to other RNAs (Zhang et al., 2013). In our study, we determined the $\mathrm{A} / \mathrm{U}$ and $\mathrm{C} / \mathrm{G}$ ratio as $50-65 \%$ and $35-50$ $\%$, respectively, which suggest the presence of more $\mathrm{U}$ and $\mathrm{C}$ nucleotides in precursor sequences. In most of the mature miRNAs sequences uracil is present at the first position expressing its important role in miRNA mediated regulation in plants. Another major criterion for determining the stability is the lower the MFE value more stable the RNA secondary structure (Bonnet et al., 2004). The MFE of the identified potato pre-miRNAs in this study was varied from 55.2 (stu-miR2673) to -338 (stu-miR395x) kcal mol ${ }^{-1}$ however, this large variation in MFE values signifies variation in their sequence length (Yang et al., 2013).

\section{Target prediction of miRNAs and functional analysis}

In miRNAs a perfect or nearly perfect match to their target mRNAs helps to regulate post-transcriptional gene expression either by endolytic cleavage of mRNA transcripts or by inhibiting translation or both (Chen, 2009). However, in plants the mismatches occurred around the middle of miRNA/mRNA complementary region which disables the cleavage activity of RISC. We predicted the potential miRNAs targets in the psRNA database using our eight miRNAs family as query sequences. The identified novel conserved miRNAs showed multiples targets in response to late blight resistance mechanism in somatic hybrid. In this study, we predicted 682 potential targets of 16 miRNAs ( 8 miRNAs families) in the potato genome database such as miR395 (29 targets), miR821 (37 targets), miR1030 (22 targets), miR1510 (113 targets), miR2673 (254 targets), miR3979 (6 targets), miR5021 (191 targets) and miR5213 (30 targets) (data not shown). These 682 potential miRNAs targets belong to several gene families with different biological functions for potato growth and development and responses to diseases and environmental stress. To illustrate selected potential targets are presented in Table 2. In particular, target genes belong to various disease resistance proteins and transcription factors categories such as NBSLRR resistance protein, Zinc finger family protein, F-box protein family, WRKY protein, Hcr2-0B and GATA transcription factor. In plants, the NBS-LRR resistance protein forms the largest class of genes and function as intracellular receptors, which code for proteins with NBSLRR domains (Bozkurt et al., 2011). The WRKY transcription factor plays key roles as repressors as well as activators in plant developmental processes, as higher expression of transcription related WRKY genes has been reported during the compatible interaction of potato and $P$. infestans (Dellagi et al., 2000). In potato, most of the chromo domains and gag-pol polyprotein containing proteins have a $\mathrm{CCHC}$ zinc finger domains playing broader functions in zinc ion and nucleic acid binding (Lawrencea et al., 2014).

Of the eight identified miRNAs families, first important miR395 family is a conserved small RNA family that has been found in Arabidopsis, rice, wheat, Brassica and many plant species. It implicates to a range of plant developmental process and abiotic/biotic stress response, as illustrated in variety of biological functions in potato (Xie et al., 2011), and leaf and stolon tissues-specific potato miRNA (Zhang et al., 2013). Moreover, miR395 family is organized into a cluster and stable miRNA395 genes expression was demonstrated in the leaf tissues of potato (Zhang et al., 2009) and Brassica oleracea (Wang et al., 2012a). Recent research has shown that miR395 family plays key role in sulfur deficiency metabolism in Arabidopsis (Liang et al., 2015), drought regulation in wheat (Akdogan et al., 2015) and arsenic-induced sulfur metabolism in Brassica juncea (Srivastava et al., 2013) to name a few. Second, miR821 family has been demonstrated in response to stress and metabolic processes in potato (Xie et al., 2011). This miR821 family is known for boron-deficiency-responsive microRNAs in Citrus sinensis roots (Lu et al., 2014), low-N tolerance in rice genotypes (Nischal et al., 2012) and play a similar regulatory mechanism in Asiatic cotton (Wang et al., 2012b). Whereas, third miR1030 family may play important roles in growth and development of Solanaceae (Kim et al., 2011), down regulated under drought stress in rice (Zhou et al., 2010), adaptive response to abiotic stress in cotton (Boopathi and Pathmanaban, 2012) and cold-responsive miRNAs in peach (Barakat et al., 2012). The fourth miR1510 family has been identified for various plant developmental process including biotic and abiotic stress response in potato (Din et al., 2014); and resistance to the soybean cyst nematode in root (Xu et al., 2014) and RNA populations of seed and vegetative tissues in soybean (Zabala et al., 2012). In another study, miR1510 family, a legume-specific family, was detected in tissue-specific organ profiles by massive-scale RNA sequencing in common bean (Peláez et al., 2012). Fifth, miR2673 family has been identified for proline accumulation under drought stress in potato (Yang et al., 2013) and plant developmental regulation in a shrub Amborella trichopoda (Hajieghrari et al., 2015). Sixth miR3979 gene family 
Table 1. Computational identification of miRNAs in potato somatic hybrid for late blight resistance.

\begin{tabular}{|c|c|c|c|c|c|c|c|c|c|c|}
\hline PGSC gene ID ${ }^{a}$ & miRNA $^{b}$ & miRNA family & miRNA sequence $\left(5^{\prime} \rightarrow 3^{\prime}\right)$ & Homologous microRNA & strand & LM (nt) & $\mathrm{AU}(\%)$ & CG $(\%)$ & MFE $(-\mathrm{kcal} / \mathrm{mol})$ & MFEI (-kcal/mol) \\
\hline PGSC0003DMG405025785 & stu-miR395x & 395 & GUGAAGUGUUUGGAGUAGCUC & osa-miR395x & minus & 20 & 64.76 & 35.24 & -338 & -0.61 \\
\hline PGSC0003DMG400019806 & stu-miR821b & 821 & AAGUUAUGAACAUAAAAGUUG & sbi-miR821b & minus & 20 & 61.63 & 38.37 & -249 & -0.53 \\
\hline PGSC0003DMG400036193 & $\begin{array}{l}\text { stu-miR1030 } \\
\quad \text { (a to g) }\end{array}$ & 1030 & UCUGCAUCUGCACCUGCACCA & ppt-miR1030a & plus & 20 & 59.21 & 40.79 & -134 & -0.57 \\
\hline PGSC0003DMG400037942 & $\begin{array}{l}\text { stu-miR } 1030 \\
\quad(\text { a to } g)\end{array}$ & 1030 & UCUGCAUCUGCACCUGCACCA & ppt-miR1030a & plus & 20 & 57.2 & 42.8 & -137 & -0.54 \\
\hline PGSC0003DMG400045212 & $\begin{array}{l}\text { stu-miR } 1030 \\
\quad(\text { a to } g)\end{array}$ & 1030 & UCUGCAUCUGCACCUGCACCA & ppt-miR1030a & plus & 20 & 50 & 50 & -64.7 & -0.51 \\
\hline PGSC0003DMG400040435 & $\begin{array}{l}\text { stu-miR } 1030 \\
\quad \text { (a to g) }\end{array}$ & 1030 & UCUGCAUCUGCACCUGCACCA & ppt-miR1030a & plus & 20 & 57.15 & 42.85 & -70.3 & -0.45 \\
\hline PGSC0003DMG400026433 & $\begin{array}{l}\text { stu-miR1510 } \\
\quad(a \text { and b) }\end{array}$ & 1510 & UGUUGUUUUACCUAUUCCACC & gso-miR1510 ( $\mathrm{a}$ and $\mathrm{b}$ ) & minus & 20 & 63.19 & 36.81 & -292 & -0.5 \\
\hline PGSC0003DMG400026433 & stu-miR5213 & 5213 & UACGUGUGUCUUCACCUCUGAA & mtr-miR5213 & minus & 21 & 63.19 & 36.81 & -292 & -0.5 \\
\hline PGSC0003DMG400038875 & $\begin{array}{l}\text { stu-miR2673 } \\
\text { (a and b) }\end{array}$ & 2673 & CCUCUUCCUCUUCCUCUUCCAC & mtr-miR2673a & minus & 21 & 54.62 & 45.38 & -55.2 & -0.48 \\
\hline PGSC0003DMG400038875 & $\begin{array}{l}\text { stu-miR3979-5p } \\
\text { stu-miR5021 }\end{array}$ & $\begin{array}{l}3979 \\
5021\end{array}$ & $\begin{array}{l}\text { UCUCUCUCUCCCUUGAAGGC } \\
\text { UGAGAAGAAGAAGAAGAAAAA }\end{array}$ & $\begin{array}{l}\text { osa-miR3979-5p } \\
\text { ath-miR5021 }\end{array}$ & $\begin{array}{l}\text { plus } \\
\text { plus }\end{array}$ & $\begin{array}{l}19 \\
19\end{array}$ & $\begin{array}{l}54.62 \\
54.62\end{array}$ & $\begin{array}{l}45.38 \\
45.38\end{array}$ & $\begin{array}{l}-59.1 \\
-59.1\end{array}$ & $\begin{array}{l}-0.52 \\
-0.52\end{array}$ \\
\hline $\begin{array}{l}\text { PGSC0003DMG400038875 } \\
\text { PGSC0003DMG400021883 }\end{array}$ & $\begin{array}{l}\text { stu-miR2673 } \\
\text { (a and b) }\end{array}$ & 2673 & CCUCUUCCUCUUCCUCUUCCAC & mtr-miR2673a & minus & 21 & 60.54 & 39.46 & -183 & -0.54 \\
\hline
\end{tabular}

${ }^{a}$ PGSC genes descriptions are available at the Potato Genome browser (http://potato.plantbiology.msu.edu/ index.shtml) as: Dynamin (PGSC0003DMG405025785); Disease resistance protein RGA3 (PGSC0003DMG400019806); Gag-pol (PGSC0003DMG400038875); and Reverse transcriptase (PGSC0003DMG400021883). GO annotation is unknown for these genes ID, except PGSC0003DMG400026433 [(GO:0007165: signal transduction) (P) and GO:0000166 (nucleotide binding) ( $\mathrm{F}) ; \mathrm{P}=$ biological process, $\mathrm{F}=$ molecular function].

stu-miR1030 (a to g): stu-miR1030a, stu-miR1030b, stu-miR1030c, stu-miR1030d, stu-miR1030e, stu-miR1030f and stu-miR1030g; stu-miR1510 (a and b): stu-miR1510a and stu-miR1510b; stu-miR2673 (a and b): stu-miR2673a and stumiR2673b.

Table 2. Selected potential targets of identified miRNAs predicted in potato somatic hybrid for late blight resistance in the potato genome.

\begin{tabular}{|c|c|c|c|}
\hline \multirow[t]{2}{*}{ miRNA family } & \multirow[t]{2}{*}{ Total number predicted } & \multicolumn{2}{|c|}{ Selected target gene } \\
\hline & & PGSC description & PGSC ID* \\
\hline \multirow[t]{2}{*}{395} & 29 & F-box protein family & PGSC0003DMT400027715 \\
\hline & & Disease resistance protein & PGSC0003DMT400017032 \\
\hline \multirow[t]{3}{*}{821} & 37 & WRKY protein & PGSC0003DMT400042399 \\
\hline & & Hcr2-0B & PGSC0003DMT400012045 \\
\hline & & Disease resistance protein & PGSC0003DMT400047509 \\
\hline \multirow[t]{2}{*}{1030} & 22 & Tir-nbs resistance protein & PGSC0003DMT400096975 \\
\hline & & Zinc finger $\mathrm{CCCH}$ domain-containing protein 13 & PGSC0003DMT400062532 \\
\hline \multirow[t]{3}{*}{1510} & 113 & NBS-coding resistance gene protein & PGSC0003DMT400067963 \\
\hline & & Disease resistance protein R3a & PGSC0003DMT400047772 \\
\hline & & Cc-nbs-lrr resistance protein & PGSC0003DMT400072165 \\
\hline \multirow[t]{3}{*}{2673} & 254 & Transcription factor & PGSC0003DMT400007015 \\
\hline & & Zinc finger family protein & PGSC0003DMT400009940 \\
\hline & & GATA transcription factor & PGSC0003DMT400024205 \\
\hline 3979 & 6 & Transcription factor TF2 & PGSC0003DMT400011408 \\
\hline \multirow[t]{3}{*}{5021} & 191 & Ring finger containing protein & PGSC0003DMT400014732 \\
\hline & & NBS-LRR resistance protein & PGSC0003DMT400019830 \\
\hline & & WRKY domain class transcription factor & PGSC0003DMT400064255 \\
\hline \multirow[t]{2}{*}{5213} & 30 & Tir-nbs-lrr resistance protein & PGSC0003DMT400053047 \\
\hline & & Disease resistance protein & PGSC0003DMT400008870 \\
\hline Total & 682 & - & - \\
\hline
\end{tabular}

Total $\frac{682}{\text { *Details of genes are available at the Potato Genome browser (http://potato.plantbiology.msu.edu/ index.shtml) }}$ 


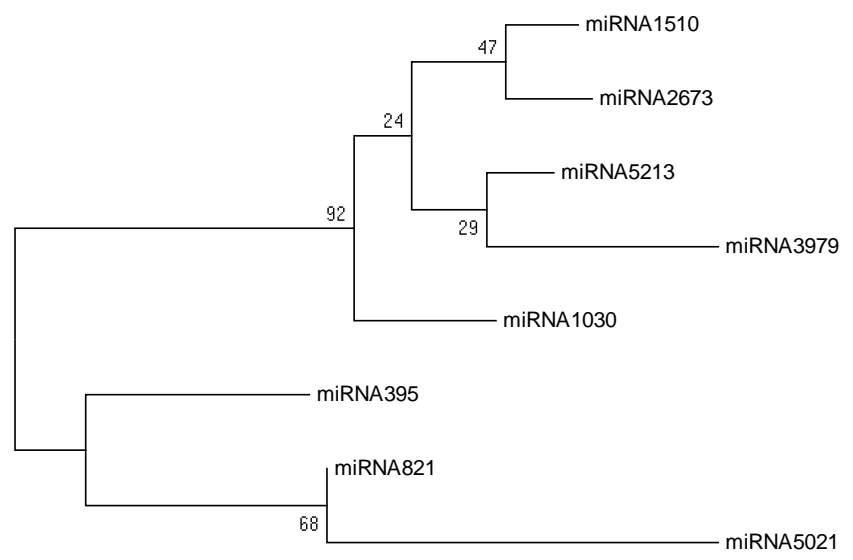

$\stackrel{\longmapsto}{0.20}$

Fig 1. Phylogenetic tree shows eight miRNAs sequence diversity based on the Neighbor-Joining method.

implicates to rice root development (Ma et al., 2013) and resistance to the blast fungus Magnaporthe oryzae in rice (Campo et al., 2013). Importantly, seventh miR5021 family is called MYB transcription factor and is a key factor in regulatory networks controlling development, metabolism and responses to biotic and abiotic stresses (Dubos et al., 2010). The miR5021 participate in multiple biological and metabolic processes, including signal transduction, stress response, MYB transcription factors and plant development in Brassica oleracea (Wang et al., 2012a) and cotton (Wang et al., 2012b). It plays important role in plant development in amino acid (cysteine and methionine) metabolism in Ocimum basilicum (Singh and Sharma, 2014) and regulates ethylene and polyamines syntheses in Catharanthus roseus (Pani and Mahapatra, 2013). Being one of the largest miRNAs families in response to cold stress, it may regulate cellular, physiological or developmental processes specific to peach and/or other tree species (Barakat et al., 2012). Lastly, degradome data in mycorrhized roots or control conditions showed that genes associated to defense responses are specifically regulated by miR5213 (Devers et al., 2011). In another study Kohli et al. (2014) identified several conserved and novel miRNAs including miR5213 in the chickpea that are associated with gene regulation following exposure to wilt and salt stress. Further, Wang et al. (2011) also identified this miR5213 in drought-responsive microRNAs in Medicago truncatula by genome-wide high-throughput sequencing.

\section{Materials and Methods}

\section{Source of potato transcripts}

The present study was performed with the microarray data of 153 (66 genes GO annotated +87 genes PGSC descriptor known) up-regulated known genes obtained in another study on gene expression profiles for late blight resistance between interspecific potato somatic hybrid (P-7) and susceptible control (C-13) (Singh et al. 2016). The plant materials for microarray were developed previously via protoplasts fusion between dihaploid Solanum tuberosum ' $\mathrm{C}-13$ ' and late blight resistant wild S. pinnatisectum (Sarkar et al., 2011). In this study, the nucleotide sequences of the 153 putative known function potato transcripts were retrieved from the potato genome browser (http://potato.plantbiology.msu.edu) for computational analyses.

\section{Availability of database and software}

To identify miRNAs, a set of plant miRNAs were compared with the 153 genes sequence of potato somatic hybrid. The tool C-mii software with complete graphical user interfaces was used to identify miRNAs in the miRBase (version miRBase Release 20) (http://microrna.sanger.ac.uk/ sequences/) consisted of mature miRNA sequences of Medicago truncatula, Physcomitrella patens, Populus trichocarpa Triticum aestivum, Aegilops tauschii, Brachypodium distachyon, Hordeum vulgare, Zea mays, Oryza sativa, Saccharum ssp., Sorghum bicolor, Brassica ssp., Arabidopsis thaliana, Glycine max, Vitis vinifera and Solanum lycopersicum. The Plant Small RNA Analysis Server (psRNATarget) was used for miRNA target analysis (Dai and Zhao, 2011) to the potato genome database. Gene description was recorded from the PGSC (http://potato.plantbiology.msu.edu/ index.shtml) and functional characterization by the GO annotation (www.geneontology.org).

\section{Identification of miRNA and prediction of secondary structure}

The tool C-mii software (http://www.biotec.or.th/isl/c-mii) was used to identify miRNAs (Numnark et al., 2012). To identify the potential conserved miRNAs in potato somatic hybrid a BLASTn search was performed with the known mature plant miRNAs from the miRBase database searched against the 153 genes sequences. During the BLASTn search no more than $3 \mathrm{nt}$ substitutions, including deletion and insertion mutations for the alignments, between known miRNAs and the homolog sequences were allowed. The screening of the candidates of potential miRNAs or premiRNAs was based on the following criteria (i) pre-miRNA sequence should fold into complete stem-loop hairpin secondary structure containing 19-22 nt mature miRNA sequence located on one arm of the hairpin structure, (ii) mature miRNAs should not have more than $3 \mathrm{nt}$ mismatches in sequence with all previously known plant mature miRNAs, (iii) the predicted secondary structures of pre-miRNA should have high MFEI and negative MFE, (iv) the A+U content should be in range of $30-70 \%$, and $(v)$ no loop or gaps in miRNA sequences was allowed. The MFEI was calculated using the equation MFEI $=[(\mathrm{MFE} /$ length of the pre-miRNA 
sequence $) \times 100] /(\mathrm{G}+\mathrm{C}) \%$. The software MFOLD3.2 was used to analyze the secondary structure of RNAs, (Zuker, 2003) (http://mfold.rna.albany.edu/?q=mfold/RNA-FoldingForm), whereas RNAfold tool (http:// rna.tbi.univie.ac.at/cgibin/RNAfold.cgi) was used to improve the efficiency of prediction of miRNAs (Hofacker, 2003). All parameters in the MFOLD were set to default values where MFE denotes the negative folding free energies.

\section{Targets prediction of miRNA targets}

The potential targets of the identified conserved miRNAs were detected using the web-server psRNATarget (http://plantgrn.noble.org/psRNATarget/?function=2) (Dai and Zhao, 2011). Where miRNAs sequence was submitted in FASTA format and searched against the Potato Genome 3.4 transcripts database (miRBase Release 20, June 2013). The parameters used for the search in psRNATarget program execution where score or maximum expectation at 3 , length of complementary scoring at 20, allowed maximum energy to unpair the target site: at 25, flanking length around target 17 bp upstream and $13 \mathrm{bp}$ downstream and keeping range of central mismatch leading to translational inhibition in between 9-11 nt.

\section{Phylogenetic Analysis of miRNA}

The phylogenetic analysis of the miRNAs was carried out using the software program Molecular Evolutionary Genetics Analysis 6 (MEGA6) (Tamura et al. 2013). The miRNA sequences were aligned by multiple sequence alignment using default parameters in the ClustalW2 tool of EMBL-EBI program. The aligned sequences data were used for the construction of the phylogenetic tree using the NeighborJoining method. The bootstrap values for the dendrogram were produced using the 'bootstrap' function of MEGA6 program with 1000 replications.

\section{Conclusion}

miRNAs play important role in many developmental and physiological processes by specifically suppressing the translation of target genes by binding to the mRNAs or degrading mRNA. The identified eight conserved miRNAs would necessarily advance our current understanding of the regulatory roles of miRNA in our interspecific potato somatic hybrid to dissect its late blight resistance mechanism. The potential target genes would considerably broaden the scope to better understanding their role in response to the pathogen. Furthermore, in vitro functional characterization of these conserved miRNAs would shed more light in understanding various pathways during host-pathogen interactions.

\section{Acknowledgements}

The authors are grateful to the ICAR-Central Potato Research Institute, Shimla, Himachal Pradesh, India (Project No. P12010/3-IPR-F30/0210) for providing necessary support to this research work.

\section{References}

Akdogan G, Tufekci ED, Uranbey S, Unver T (2015) miRNA-based drought regulation in wheat. Funct Integr Genomics. DOI: 10.1007/s10142-015-0452-1.
Barakat A, Sriram A, Park J, Zhebentyayeva T, Main D, Abbott A (2012) Genome wide identification of chilling responsive microRNAs in Prunus persica. BMC Genomics. 13:481.

Bonnet E, Wuyts J, Rouze P, van de Peer Y (2004) Evidence that microRNA precursors, unlike other non-coding RNAs, have lower folding free energies than random sequences. Bioinformatics. 20:2911-2917.

Boopathi NM, Pathmanaban R (2012) Additional insights into the adaptation of cotton plants under abiotic stresses by in silico analysis of conserved miRNAs in cotton expressed sequence tag database (dbEST). Afr J Biotech. 11:1405414063.

Bozkurt TOS, Schornack J, Win T, Shindo M, Ilyas R, Olivaa L, Canoa M, Jonesa AME, Huitemaa E, van der Hoornb RAL, Kamouna S (2011) Phytophthora infestans effector AVRblb2 prevents secretion of a plant immune protease at the haustorial interface. Proc Nat Acad Sci USA. 108:20832-837.

Campo S, Peris-Peris C, Sire C, Moreno AB, Donaire L, Zytnicki M, Notredame C, Llave C, Segundo BS (2013) Identification of a novel microRNA (miRNA) from rice that targets an alternatively spliced transcript of the Nramp6 (Natural resistance-associated macrophage protein 6) gene involved in pathogen resistance. New Phytologist. 199:212-227.

Chen X (2009) Small RNAs and their roles in plant development. Annu Rev Cell Dev Biol. 25:21-44.

Dai X, Zhao PX (2011) psRNATarget: a plant small RNA target analysis server. Nucleic Acids Res. 39:W155-W159.

Dellagi A, Heilbronn J, Avrova AO, Montesano M, Palva TE, Stewart HE, Toth IK, Cooke DEL, Lyon GD, Birch PRJ (2000) A potato gene encoding a WRKY-like transcription factor is induced in interactions with Erwinia carotovora subsp. atroseptica and Phytophthora infestans and is coregulated with a class I endochitinase expression. Mol Plant-Microbe Inter. 13:1092-1101.

Devers EA, Branscheid A, May P, Krajinski F (2011) Stars and symbiosis: microRNA- and microRNA*-mediated transcript cleavage involved in arbuscular mycorrhizal symbiosis. Plant Physiol. 156:1990-2010.

Din M, Barozai MYK, Baloch IA (2014) Identification and functional analysis of new conserved microRNAs and their targets in potato (Solanum tuberosum L.). Turk J Bot. 38:1199-1213.

Dubos C, Stracke R, Grotewold E, Weisshaar B, Martin C, Lepiniec L (2010) MYB transcription factors in Arabidopsis. Trends Plant Sci. 15:573-581.

Hajieghrari B, Farrokhi N, Goliaei B, Kavousi K (2015) Computational identification, characterization and analysis of conserved miRNAs and their targets in Amborella Trichopoda. J Data Mining Genomics Proteomics. 6:2.

Hofacker IL (2003) Vienna RNA secondary structure server. Nucleic Acids Res. 31:3429-3431.

Kim HJ, Baek KH, Lee BW, Choi D, Hur CG (2011) In silico identification and characterization of microRNAs and their putative target genes in Solanaceae plants. Genome. 54:9198.

Kohli D, Joshi G, Deokar AA, Bhardwaj AR, Agarwal M, Agarwal SK, Srinivasan R, Jain PK (2014) Identification and characterization of wilt and salt stress-responsive microRNAs in chickpea through high-throughput sequencing. PLoS One. 9:e108851.

Lawrencea SD, Novaka NG, Jones RW, Jr RRF, Blackburna MB (2014) Herbivory responsive $\mathrm{C} 2 \mathrm{H} 2$ zinc finger transcription factor protein StZFP2 from potato. Plant Physiol Biochem. 80:226-233. 
Liang G, Ai Q, Yu D (2015) Uncovering miRNAs involved in crosstalk between nutrient deficiencies in Arabidopsis. Scientific Reports. 5:11813.

Lu Y-B, Yang L-T, Qi Y-P, Li Y, Li Z, Chen Y-B, Huang Z$R$, Chen L-S (2014) Identification of boron-deficiencyresponsive microRNAs in Citrus sinensis roots by Illumina sequencing. BMC Plant Biol. 14:123.

Luo X, Gao Z, Shi T, Cheng Z, Zhang Z, Ni Z (2013) Identification of miRNAs and their target genes in peach (Prunus persica L.) using high-throughput sequencing and degradome analysis. PLoS One. 8:e79090.

Ma X, Shao C, Wang H, Jin Y, Meng Y (2013) Construction of small RNA-mediated gene regulatory networks in the roots of rice (Oryza sativa). BMC Genomics. 14:510.

Nischal L, Mohsin M, Khan I, Kardam H, Wadhwa A, Abrol YP, Iqbal M, Ahmad A (2012) Identification and comparative analysis of microRNAs asociated with low-N tolerance in rice genotypes. PLoS One. 7:e50261.

Numnark S, Mhuantong W, Ingsriswang S, Wichadakul D (2012) C-mii: a tool for plant miRNA and target Identification. BMC Genomics. 13(Suppl 7):S16.

Olsen PH, Ambros V (1999) The lin-4 regulatory RNA controls developmental timing in Caenorhabditis elegans by blocking LIN-14 protein synthesis after the initiation of translation. Dev Biol. 216:671-680.

Pani A, Mahapatra RK (2013) Computational identification of microRNAs and their targets in Catharanthus roseus expressed sequence tags. Genomics Data. 1:2-6.

Park W, Li J, Song R, Messing J, Chen X (2002) CARPEL FACTORY, a Dicer homolog, and HEN1, a novel protein, act in microRNA metabolism in Arabidopsis thaliana. Current Biol. 12:1484-1495.

Peláez P, Trejo MS, Iñiguez LP, Estrada-Navarrete G, Covarrubias AA, Reyes JL, Sanchez F (2012) Identification and characterization of microRNAs in Phaseolus vulgaris by high-throughput sequencing. BMC Genomics. 13:83.

Sarkar D, Tiwari JK, Sharma SH, Poonam, Sharma SA, Gopal J, Singh BP, Luthra SK, Pandey SK, Pattanayak D (2011) Production and characterization of somatic hybrids between Solanum tuberosum L. and S. pinnatisectum Dun. Plant Cell Tiss Organ Cult. 107:427-440.

Singh N, Sharma A (2014) In-silico identification of miRNAs and their regulating target functions in Ocimum basilicum. Gene. 552:277-282.

Singh R, Tiwari JK, Rawat S, Sharma V, Singh BP (2016) Monitoring gene expression pattern in somatic hybrid of Solanum tuberosum and $S$. pinnatisectum for late blight resistance using microarray analysis. Plant Omics. 9:99105.

Srivastava S, Srivastava AK, Suprasanna P, D'Souza SF (2013) Identification and profiling of arsenic stress-induced microRNAs in Brassica juncea. J Exp Bot. 64:303-315.
Tamura K, Stecher G, Peterson D, Filipski A, Kumar S (2013) MEGA6: Molecular Evolutionary Genetics Analysis Version 6.0. Mol Biol Evol. 30:2725-2729.

Tiwari JK, Devi S, Sharma S, Chandel P, Rawat S, Singh BP (2015) Allele mining in Solanum germplasm: cloning and characterization of RB-homologous gene fragments from late blight resistant wild potato species. Plant Mol Biol Rep. 33:1584-1598.

Wang J, Yang X, Xu H, Chi X, Zhang M, Hou X (2012a) Identification and characterization of microRNAs and their target genes in Brassica oleracea. Gene. 505:300-308.

Wang M, Wang Q, Wang B (2012b) Identification and characterization of microRNAs in Asiatic cotton (Gossypium arboreum L.). PLoS One. 7:e33696.

Wang T, Chen L, Zhao M, Tian Q, Zhang W-H (2011) Identification of drought-responsive microRNAs in Medicago truncatula by genome-wide high-throughput sequencing. BMC Genomics. 12:367.

Xie F, Frazier TP, Zhang B (2011) Identification, characterization and expression analysis of MicroRNAs and their targets in the potato (Solanum tuberosum). Gene. 473:8-22.

Xu M, Li Y, Zhang Q, Xu T, Qiu L, Fan Y, Wang L (2014) Novel miRNA and phasiRNA biogenesis networks in soybean roots from two sister lines that are resistant and susceptible to SCN race 4. PLoS One. 9:e110051.

Yang J, Zhang N, Ma C, Qu Y, Si H, Wang D (2013) Prediction and verification of microRNAs related to proline accumulation under drought stress in potato. Comput Biol Chem. 46:48-54.

Zabala G, Campos E, Varala KK, Bloomfield S, Jones SI, Win H, Tuteja JH, Calla B, Clough SJ, Hudson M, Vodkin LO (2012) Divergent patterns of endogenous small RNA populations from seed and vegetative tissues of Glycine max. BMC Plant Biol. 12:177.

Zhang R, Marshall D, Bryan GJ, Hornyik C (2013) Identification and characterization of miRNA transcriptome in potato by high throughput sequencing. PLoS One. 8:e57233.

Zhang W, Luo Y, Gong X, Zeng W, Li S (2009) Computational identification of 48 potato microRNAs and their targets. Comput Biol Chem. 33:84-93.

Zhou L, Liu Y, Liu Z, Kong D, Duan M, Luo L (2010) Genome-wide identification and analysis of droughtresponsive microRNAs in Oryza sativa. J Exp Bot. 61:4157-416

Zuker M (2003) Mfold web server for nucleic acid folding and hybridization prediction. Nucleic Acids Res. 31:34063415 . 